\title{
Models and Techniques for Computer Aided Surgical Training
}

\author{
Jerzy W. Rozenblit \\ Dept. of Electrical and Computer Engineering \\ Dept. of Surgery \\ The University of Arizona,Tucson, Arizona 85721, USA \\ jr@ece.arizona.edu
}

Keywords: minimally invasive surgery, computer-guided surgery, virtually assisted training.

\section{Introduction}

Laparoscopic surgery, when performed by a well-trained surgeon, is a remarkably effective procedure that minimizes complications associated with large incisions, operative blood loss and post-operative pain. It also reduces the recovery time. However, the procedure is more challenging than a conventional surgery due to the restricted vision, hand-eye coordination problems, limited working space, and lack of tactile sensation. These issues make the laparoscopic surgery a more difficult technique for medical students and residents to master.

An effective training environment must provide high fidelity and repeatable exercises that are well structured to meet specific learning objectives. Analogies to the aviation world are often used as a strong motivating factor. Just as no pilot can be certified to fly a particular aircraft unless he or she has completed simulated cockpit training, no surgeon should attempt laparoscopic procedures on a patient without extensive virtual training.

Gallagher [1] posits that the goal of any surgical training program is to help surgeons automate their basic psychomotor skills before they operate on a patient (,,the more innate visuospatial, perceptual, and psychomotor ability the surgeon has, the faster he or she will automate the surgical skills").

One class of simulation-based training tools is called the virtual reality simulators (VRS) [4], 13], 16], 22], 23], 25, 26]. VRS systems use a computer to simulate the entire training procedure. Trainees interact with the simulator through a specially designed interface. VRS simulations often provide inadequate perception of reality and inaccurate haptic feedback. Those limitations make the performance of a VRS as a training tool questionable [24, 26].

A number of physicians prefer to use another type of a tool called the pelvic trainer. A pelvic trainer is just a box with apertures that simulates the abdomen. Trainees use real instruments to practice basic skills and observe the operating scene through a video display. The trainer provides a degree of realism and some haptic feedback. It is orders of magnitude less expensive that the VRS systems. The main limitation of this approach is the absence of an

R. Moreno-Díaz et al. (Eds.): EUROCAST 2011, Part II, LNCS 6928, pp. 233-241, 2012.

(C) Springer-Verlag Berlin Heidelberg 2012 
objective performance assessment. The only quantitative measurement device used frequently in the educational and clinical research is the stopwatch [12. In the recent decade, a great number of surgical skills training tools have become commercially available that fall into the two above specified classes. We refer the reader to [4], [5], 6], [7], [8], [9], [14, , 10, , 18, ,25] for a review of some of the existing systems.

In our work, we focus on ,,computer-assisted surgical training” (CAST). Our design principles and their subsequent implementation address some of the limitations of the existing systems and advance the state of the art in surgical education, assessment, and guidance in laparoscopic surgery [4, [6], [7, , 8], [9], 21, [27], 28. Our overarching vision is to develop a fully integrated training system that will serve as a ,,cognitive amplifier" for a practicing surgeon. Such a system will enhance training and provide assistance in real-time during an operation. Our goal is to help improve surgical outcomes and patients safety.

In this chapter, a high-level overview of our activities is given with appropriate references to various stages of CAST development. We begin with the overall design concept and its elements.

\section{Design Concept and Implementation}

Our CAST design concept was driven by the need to simulate surgical procedures in stages, represent anatomical variations and anomalies, permit random introduction of unforeseen crises, and to provide haptic feedback. The system should have methods and tools that track and assess trainees performance.

In [5, 6], 7], 8], we defined three fundamental design layers for CAST. Layer 1, called the Perception Layer embodies physical sensing devices, tracking (motion, touch accuracy, etc.) and detection algorithms. The key driving need here was to design and implement the ability to precisely track the position of surgical instruments during a training session. This allows us and the trainees to review their performance with respect to a set of metrics such as the economy of movement, time, accuracy, direction profile, etc. [6], 21. This led directly to the second design layer, namely the Comprehension Layer, which provides a suite of metrics and algorithms for objective performance assessment.

We had commenced the initial (CAST I) system design and development by equipping surgical instruments with magnetic sensors Micro Bird [2] for precise tracking and data collection. The position data obtained from the Micro Bird sensors is used to calculate key instrument motion metrics such as total path length, average speed, instantaneous speed, average radius of motion and number of times ,,safety zones" were breached. Using this concept, we conducted a study [5], comparing the efficacy of a standard surgical monitor, a high definition (HD), display, and stereoscopic (3 dimensional 3D) display system.

The results of the study indicated that while the higher resolution provided by HD displays was widely favored by surgeons, it did not yield significant improvements on parameters such as the speed and movement economy. HD displays may, in fact, have detrimental effects on such parameters when compared to 
standard lower resolution laparoscopic or stereoscopic display systems. The 3D system was generally favored by inexperienced trainees but presented ergonomic issues [5].

Furthermore, we have refined the CAST I system to allow the trainees to work with two instruments and random "targets" for tasks such as grasping. A study was conducted to address hand-dominance in two-instrument exercises. In this study, participants were asked to grasp targets correctly using minimally-invasive surgical equipment (Karl Storzß)).

There were a total of nine targets and one start point constructed out of LEGOß" bricks" for easy reconfiguration (see Figure 11). All targets had an embedded in them a metal hook surrounded by three LEDs (green, red, and yellow). The computer randomly selected a target to light up one of its LEDs. Participants were required to use the correct instrument (right instrument for the green light, left one for the red light, and both instruments for the yellow LED) to grasp the targets metal hook within ten seconds. Electromagnetic sensors (MicroBird) were used to track the instruments movements in the $3 \mathrm{D}$ space at $60 \mathrm{~Hz}$.

We evaluated participants performance metrics based on three variables: movement economy, time taken for completion and accuracy.

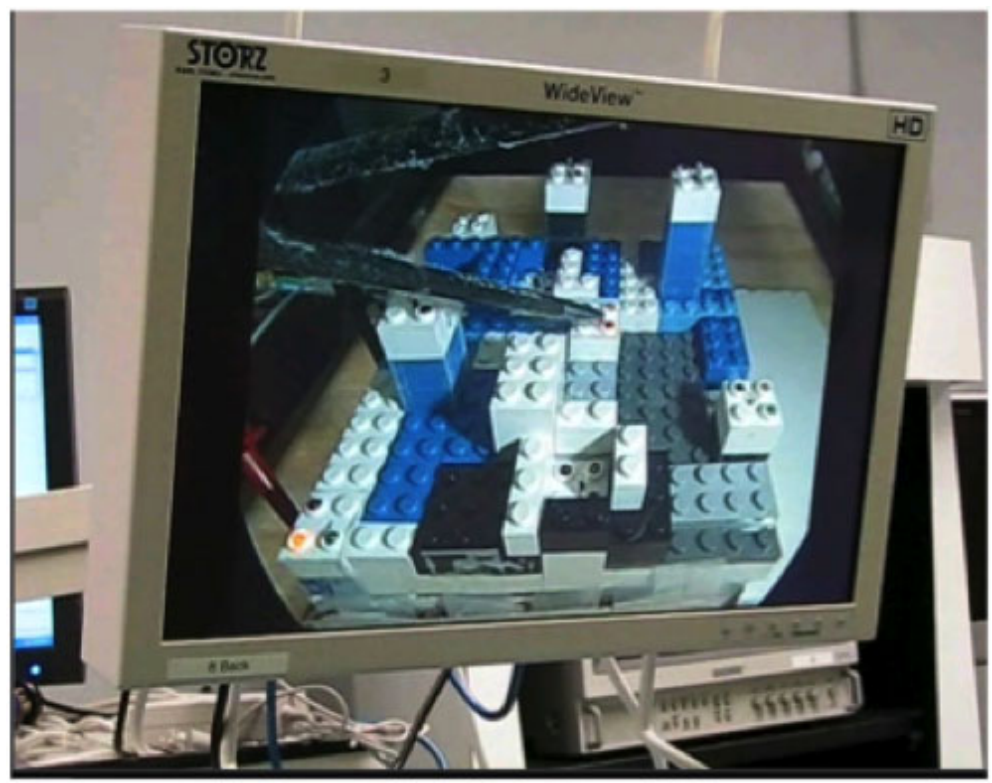

Fig. 1. Set up for hand-dominance study

Thirty subjects participated in our study. We conducted data analysis for three group variables: dominant hand vs. non-dominant hand performance, gender effect, and previous laparoscopic training experience effect. Our initial results were in favor of the non-dominant hand, and participants who had previous laparoscopic training experience. 
Our initial hypothesis was that the dominant hand performed better than the non-dominant one. However, our study disapproved the initial hypothesis as illustrated in Table 1 .

Table 1. Hand dominance results

\begin{tabular}{|c|c|c|}
\hline & Dominant & Non dominant \\
\hline $\begin{array}{c}\mathrm{ME} \\
\bar{x} \\
( \pm \mathrm{S} . \mathrm{E})\end{array}$ & $\begin{array}{r}0.5453 \\
( \pm 0.0096)\end{array}$ & $\begin{array}{r}0.5688 \\
( \pm 0.0096)\end{array}$ \\
\hline $\begin{array}{c}\mathrm{P} \\
\text { value }\end{array}$ & $\mathrm{p}<0.0001$ & $\mathrm{p}<0.0001$ \\
\hline $\begin{array}{c}\text { TTC } \\
\bar{x} \\
( \pm \mathrm{S} . \mathrm{E})\end{array}$ & $\begin{array}{r}0.6797 \\
( \pm 0.010)\end{array}$ & $\begin{array}{r}0.6753 \\
( \pm 0.010)\end{array}$ \\
\hline $\begin{array}{c}\mathrm{P} \\
\text { value }\end{array}$ & $\mathrm{p}<0.4591$ & $\mathrm{p}<0.4591$ \\
\hline $\begin{array}{c}\mathrm{PRO} \\
\bar{x} \\
( \pm \mathrm{S} . \mathrm{E})\end{array}$ & $\begin{array}{r}0.3743 \\
( \pm 0.0099)\end{array}$ & $\begin{array}{r}0.3867 \\
( \pm 0.0099)\end{array}$ \\
\hline $\begin{array}{c}\mathrm{P} \\
\text { value }\end{array}$ & $\mathrm{p}<0.0464$ & $\mathrm{p}<0.0464$ \\
\hline
\end{tabular}

In the table above, ME stands for normalized movement economy expressed at the ratio of actual path/ideal path, TTC is a completion coefficient (fast movements have a higher coefficient), PRO is the aggregate measure of proficiency expressed as the product of $M E x T C C x$ Accuracy (either 1, or 0 if the target was not grasped within 10 seconds).

We believe that the relationship between hand dominance and laparoscopic performance seems to be task related. Additional research using more complex surgical tasks is needed for clarification.

In the Comprehension Layer, we also developed the ability to assess trainees performance not only quantitatively but also qualitatively. In [18, we presented the knowledge elicitation process to model the performance metrics and the rules involved in the assessment of minimally invasive surgical skills. Our assessment model is based on fuzzy logic, so that it is easier to mimic the judgment that is already performed by experienced surgeons in qualitative terms. An empirical study to validate our approach is described in [18.

The highest, most complex element of our system, is the Projection Layer. Here, we work on implementing knowledge-based reasoning as well as real-time instrument guidance. We explain this concept in the following section.

\subsection{Collision Free Guidance}

We had further enhanced the surgical trainer with real-time guidance and navigation capabilities. This version, called CAST II, employs sensing and configuration space methods [1, 3], 17] to assist in training with a special focus on 
proper execution of movements and avoidance of critical zones in the operating space. An inference module is employed to determine if a particular action is potentially harmful and the reasons why the action could be harmful. Then, guidance and feedback to prevent potentially injurious actions and to reinforce correct techniques are given to the trainee. Proper guidance includes displaying the estimated optimal path and performance instructions on the screen to help the trainees know what to do, and what not to do. This concept is demonstrated in Figure 2 which depicts one of the views available in the system showing instrument tracks, and the ,no-fly zone" (a spatial region that must not be entered by an instrument) breaches. Intrusion into ,,no-fly zones" would trigger an alert.

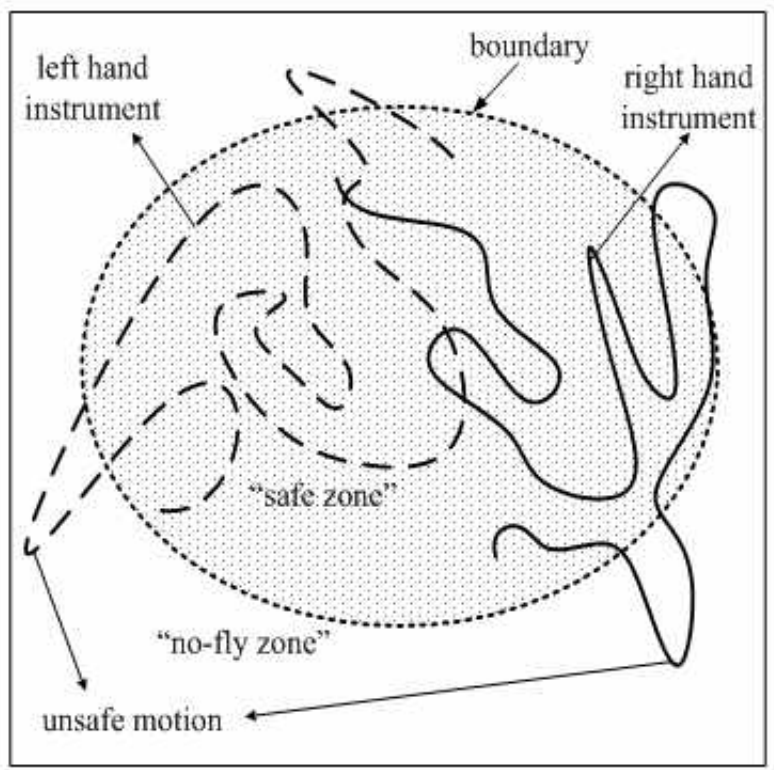

Fig. 2. "No-fly zone" diagram

In our initial study [7, [27, we used the configuration space ( $C$-space) techniques to generate the estimated optimal path of the instruments [1, [17. The first step was to model the instruments in their configuration space. As published in detail in [7, we considered the problem of a rigid instrument $A$ moving in a Euclidean space $W=R^{3}$, equipped with a fixed Cartesian coordinate system, denoted by $F_{W}$. We also represented a moving coordinate system $F$ attached to $A$ so that each point in the instrument has consistent coordinates in $F$ (Figure 3).

The origin of $F_{W}$ is $O_{W}$, and the origin of $F_{A}$ is $O_{A} . O_{A}$ is the reference point of $A$.

The configuration space of $A$ is the space $C$ of all the possible positions of $A$ , subject to external constraints. Now, suppose $W$ contains a series of physical 


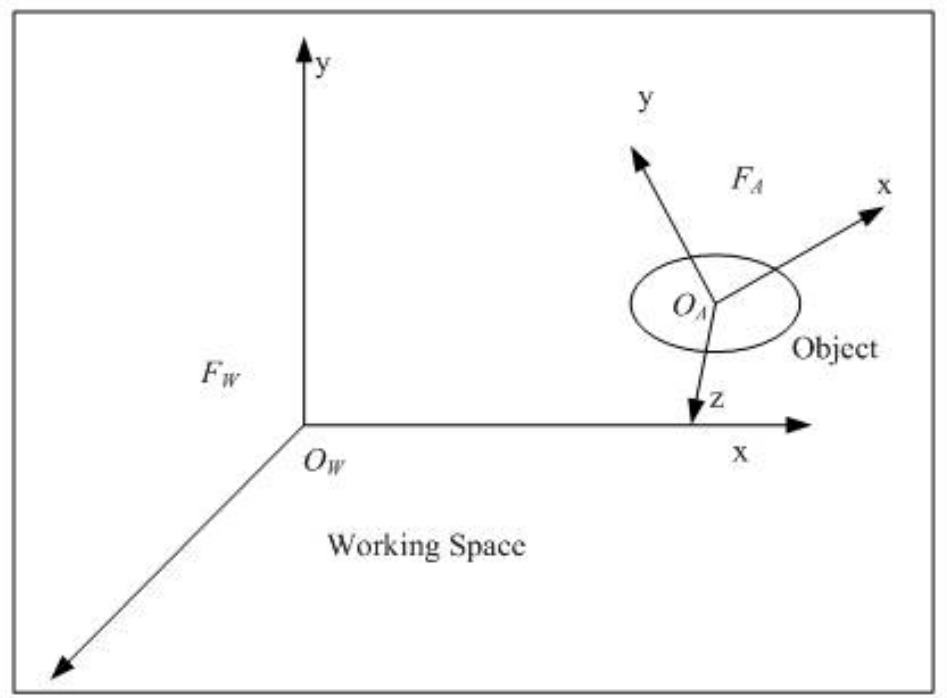

Fig. 3. Working space and object coordinates

obstacles $B_{i}, i=1,2, \ldots, q$. Each obstacle $B_{i}$ in $W$ maps in $C$ space to the region $C B_{i}$, which is called a C-obstacle.

In our application, the main task was to define the C-obstacles. There are several different kinds of C-obstacles in the current CAST system. The first one is the safety space boundary; the second one are the objects within the safety boundary; the third one is the opposite instrument; and the fourth one is the additional constraints.

We are presently focusing on the optimal motion planning method for minimally invasive surgery (optMIS) which is aimed at searching the shortest collisionfree paths for laparoscopic instruments in 3D space with multiple ,no-fly zones". The proposed method divides the problem into path searching and path following sub-problems. The path searching stage is used to generate the shortest path for each of the instruments to avoid breaching ,,no-fly zones". In order to avoid collisions between the instruments, the velocity vectors are assigned to optimal paths at the path following stage. The optMIS method combines the advantages of computational geometry-based techniques, Dijkstras algorithm and evolutionary computation [19, 20].

\section{Next Generation Trainer}

The most likely source for a quantum leap in surgical technique is robotics. While technical and cost limitations constrain our ability to realistically 
perform surgery in this manner (i.e., robotically) now, the future may be markedly different. Currently, some haptic feedback devices have been introduced in virtual reality laparoscopic trainer systems. The basic idea of these devices is connecting a small robotic manipulator to surgical instruments. Therefore, force and torque can be exerted on the devices through a manipulator. The ability to link haptic devices with CAST could assist in the development of so-called "smart" instruments. "Smart" instruments are much cheaper and simpler than surgical robots.

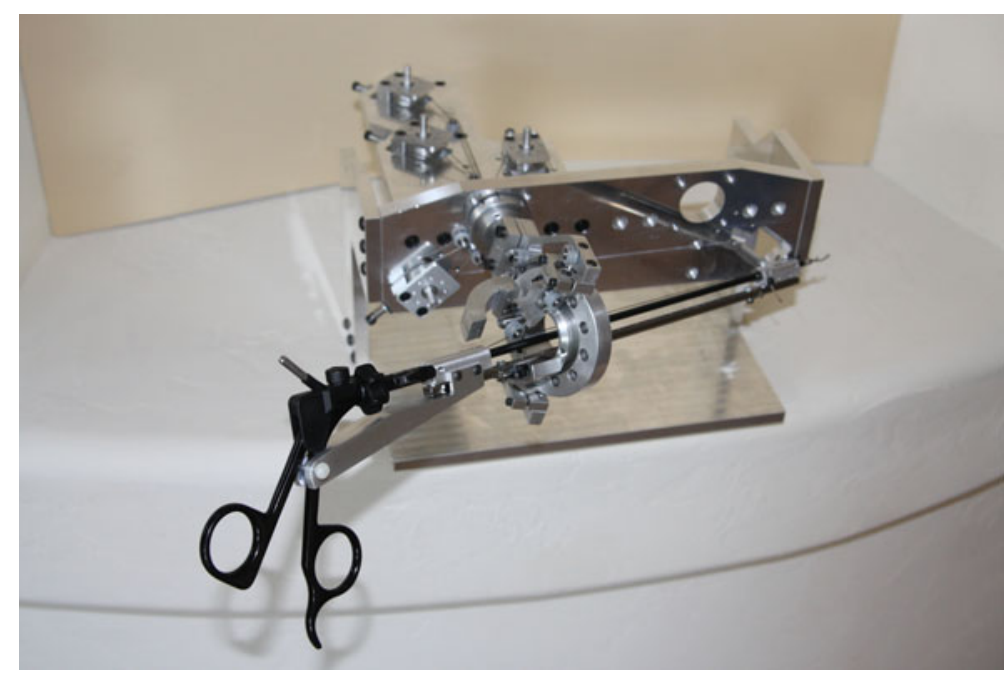

Fig. 4. CAST III device

We are developing the 3rd generation CAST system which will realize this concept (it is shown in Figure 4). It is in principle a mechatronic device that employs real surgical instruments, encoders for precise position sensing, servomotors and attendant software for motion control. We are currently completing the development of the CAST III prototype. CAST III will have embedded in it collision free and optMIS navigation.

\section{Summary}

In developing CAST, we take a dual position on the ultimate utility of our concept and its implementation. The foundational work focuses on providing training and assessment capabilities in a manner that is affordable, realistic, and objective. The ultimate vision behind our efforts is to transfer the technology or parts thereof to the operating room to provide computer-guided assistance in real-time. 


\section{References}

1. Alterovitz, R., Goldberg, K.: Motion Planning in Medicine: Optimization and Simulation Algorithms for Image-Guided Procedures. Springer, Heidelberg (2008)

2. Ascension Technology Co., microBIRD Technical Reference Guide (2005), http://www.ascension-tech.com/

3. Cohen, J., Lin, M.C., Manocha, D., Ponamgi, M.: I-Collide: An interactive and exact collision detection system for large-scale environments. In: 1995 Proceedings of ACM I3D, pp. 189-196 (1995)

4. Dankelman, J.: Surgical robots and other training tools in minimally invasive surgery. In: 2004 IEEE International Conference on Systems, Man and Cybernetics, pp. 2459-2464 (2004)

5. Falk, V., Mintz, D., et al.: Influence of three-dimensional vision on surgical telemanipulator performance. Surgical Endoscopy 15, 1282-1288 (2001)

6. Feng, C., Rozenblit, J.W., Hamilton, A.J.: A computerized assessment to compare the impact of standard, stereoscopic and high definition laparoscopic monitor displays on surgical technique. Surgical Endoscopy 24(11), 2743-2748 (2010), doi:10.1007/s00464- 010-1038-6

7. Feng, C., Rozenblit, J.W., Hamilton, A.J., Wytyczak-Partyka, A.: Defining Spatial Regions in Computer-assisted Laparoscopic Surgical Training. In: Proc. of the 16th IEEE Intl. Conference and Workshops on the Engineering of Computer Based Systems (ECBS 2009), San Francisco, pp. 176-183 (April 2009)

8. Feng, C.: A Situational Awareness Enhancing System for Minimally Invasive Surgery Training, Ph.D. Dissertation (2007)

9. Feng, C., Rozenblit, J.W., Hamilton, A.J.: A Hybrid View in a Laparoscopic Surgery Training System. In: Proc. of the 14th IEEE Intl. Conference and Workshops on the Engineering of Computer Based Systems (ECBS 2007), Tucson, Arizona, pp. 339-348 ( March 2007)

10. Feng, C., Haniffa, H., et al.: Surgical training and performance assessment using a motion tracking system. In: Proc. of the 2nd European Modeling and Simulation Symposium, EMSS 2006, October 4-6, pp. 647-652. Piera, LogiSim (2006)

11. Gallagher, A.G., McClure, N., et al.: Virtual reality training in laparoscopic surgery: a preliminary assessment of minimally invasive surgical trainer virtual reality (MIST VR). Endoscopy 31(4), 310-313 (1999)

12. Hagiike, M., Phillips, E.H., Berci, G.: Performance differences in laparoscopic surgical skills between true high-definition and three-chip CCD video system. Surgical Endoscopy 21, 1849-1854 (2007)

13. Hamilton, E.C., Scott, D.J., et al.: Comparison of video trainer and virtual reality training systems on acquisition of laparoscopic skills Journal Surgical Endoscopy. Surgical Endoscopy Journal 16, 406-411 (2002)

14. Haniffa, H., Rozenblit, J.W., Peng, J., Hamilton, A.J., Salkini, M.: Motion Planning System for Minimally Invasive Surgery. In: Proc. of the 14th IEEE Intl. Conference and Workshops on the Engineering of Computer Based Systems (ECBS 2007), Tucson, Arizona, pp. 609-610 (March 2007)

15. Hasson, H.M., Kumari, N.V., Eekhout, J.: Training simulator for developing laparoscopic skills. Journal of the Society of Laparoendoscopic Surgeons 5(3), 255-265 (2001)

16. Korndorffer Jr, J.R., Dunne, J.B., Sierra, R., et al.: Simulator training for laparoscopic suturing using performance goals translates to the operating room. J. Am. Coll. Surg. 201, 23-29 (2005) 
17. Latombe, J.: Robot Motion Planning. Kluwer Academic Publishers, Norwell (1991)

18. Leonard, J.: Sensor Fusion for Surgical Applications. In: The 15th AESS/IEEE Dayton Section Symposium, pp. 37-44 (1998)

19. Napalkova, L.: Development and Application of Multi-Objective Simulation-Based Optimisation Methods. PhD Dissertation. Riga Technical University (2010)

20. Noto, M., Sato, H.: A Method for the Shortest Path Search by Extended Dijkstra Algorithm. In: IEEE International Conference on Systems, Man and Cybernetics, pp. 2316-2320 (2000)

21. Riojas, M., Feng, C., Hamilton, A.J., Rozenblit, J.W.: Knowledge Elicitation for Performance Assessment in a Computerized Surgical Training System. Applied Soft Computing 11(4), 3697-3708

22. Shrivastava, S., Sudarshan, R., et al.: Surgical Training and Performance Evaluation using Virtual Reality based Simulator. In: Virtual Concept 2003, France (2003)

23. Simbionix Co. (2007), http://www.simbionix.com

24. Spillane, L.L., Spencer, M., Maddow, C.: Simulation a Valuable Teaching Tool or a Waste of Time (Abstract). Academic Emergency Medicine 11(5), 561 (2004)

25. Stylopoulos, N., Cotin, S., et al.: Computer-enhanced laparoscopic training system (CELTS). Surgical Endoscopy 18, 782-789 (2004)

26. Waxberg, S.L., Goodell, K.H., et al.: Evaluation of Physical Versus Virtual Surgical Training Simulators. In: Human Factors and Ergonomics Society Annual Meeting Proceedings, Medical Systems and Rehabilitation, pp. 1675-1679(5) (2004)

27. Wytyczak-Partyka, A., Nikodem, J., Klempous, R., Rozenblit, J.: A novel interaction method for laparoscopic surgery training. In: Proceedings of the 8th Annual IEEE International Conference and Workshops on Human Systems Interactions, pp. 858-861 (2008)

28. Wytyczak-Partyka, A., Nikodem, J., Klempous, R., Rozenblit, J., Feng, C.: Computer-guided laparoscopic training with application of a fuzzy expert system. In: Gelbukh, A., Morales, E.F. (eds.) MICAI 2008. LNCS (LNAI), vol. 5317, pp. 965-972. Springer, Heidelberg (2008) 